\title{
Factores determinantes y coste económico del absentismo de pacientes en consultas externas de la Agencia Sanitaria Costa del Sol
}

\author{
Determinants and economic cost of patient absenteeism in outpatient \\ departments of the Costa del Sol Health Agency
}

\author{
M.L. Jabalera Mesa' ${ }^{1}$, J.M. Morales Asencio², F. Rivas Ruiz ${ }^{3}$
}

\section{RESUMEN}

Fundamento. Identificar los factores de la persona, el proceso y el contexto que influyen en el cumplimiento de las citas de consultas externas en la Agencia Sanitaria Costa del Sol, describiendo el perfil del paciente que no acude a su cita, los motivos del absentismo y realizando una estimación del coste económico.

Método. Estudio observacional, multicéntrico de casos y controles, mediante encuesta a pacientes citados en consultas ambulatorias durante 2013 y 2014 y análisis de costes por consultas.

Resultados. Participaron en el estudio 882 pacientes (294 casos y 588 controles). El olvido ( $29,6 \% ; n=87)$ y el fallo en la comunicación $(16 \%$; $n=47)$, fueron los principales motivos manifestados por los pacientes absentistas. Una menor demora en la cita y la edad avanzada se asociaban significativamente con menos ausencias, al igual que haber frecuentado más las consultas en el último año. El coste económico fue superior a 3 millones de euros para una tasa de inasistencia del $13,8 \%$.

Conclusiones. Los pacientes jóvenes que no frecuentan habitualmente las consultas constituyen el grupo de mayor riesgo de ausencia a las citas programadas en nuestra área sanitaria. Los principales motivos del absentismo son evitables y podrían beneficiarse de intervenciones como la mejora de los procedimientos de comunicación o sistemas de recordatorio de citas.

Palabras clave. Citas y horarios. Consulta. Pacientes ambulatorios. Atención ambulatoria. Cooperación del paciente.

\begin{abstract}
Background. To identify factors related to the people, the process and the context that determine patient compliance with their appointments in the ambulatory outpatient services in the Costa del Sol Health Care Agency; and to obtain the profile of patients who fail to keep their appointments, the reasons for this failure and an estimation of its economic cost.
\end{abstract}

Methods. Observational multicenter case-control study, through a survey carried out on patients with an appointment in the ambulatory outpatient services during 2013 and 2014, and analysis of the cost per appointment.

Results. In total, 882 patients participated in the study (294 cases and 588 controls). The main reasons for missing an appointment were forgetting about it $(29$, $6 \% ; n=87)$, and failure in communication $(16 \% ; n=47)$. A shorter time period before the appointment and older age were significantly associated with fewer absences, as well as the fact of having attended more consultations in the past year. The economic cost was more than 3 million euros for a non-attendance rate of $13.8 \%$.

Conclusions. Young patients who usually do not visit outpatient clinics are at greatest risk of absence from scheduled appointments in our health area. The main reasons for absenteeism are preventable and interventions such as improved communication procedures or appointment reminder systems could be beneficial.

Keywords. Appointment and schedules. Referral and consultation. Outpatients. Ambulatory care. Patient compliance.
1. Agencia Sanitaria Costa del Sol.

2. Facultad de Ciencias de la Salud. Universidad de Málaga.

3. Red de Investigación en Servicios de Salud en Enfermedades Crónicas (REDISSEC)

Recepción: 23 de febrero de 2015

Aceptación provisional: 31 de marzo de 2015

Aceptación definitiva: 15 de abril de 2015

\section{Correspondencia:}

$M^{a}$ Lourdes Jabalera Mesa

Agencia Sanitaria Costa del Sol

Centro de Alta Resolución de Especialidades

de Mijas (CARE)

29650 Mijas (Málaga)

E-mail: mljmesa@hcs.es 


\section{INTRODUCCIÓN}

La inasistencia de los pacientes a sus citas programadas en consultas externas representa un problema en las organizaciones sanitarias, pues supone una infrautilización de los recursos humanos y materiales destinados a dicha asistencia, un esfuerzo adicional en la reprogramación de citas, un coste de oportunidad en los recursos y servicios destinados, y posibles implicaciones en la salud de los sujetos absentistas. El impacto del absentismo es más relevante cuando resulta posible la solicitud ilimitada de nuevas citas, lo que es frecuente en sistemas públicos de salud en los que no existen mecanismos reguladores de la demanda ${ }^{1}$.

Los pacientes absentistas generan ineficiencias productivas y económicas, cuyas consecuencias van desde el aumento de las listas de espera, la disminución de la accesibilidad y el incremento de la morbilidad, hasta ocasionar un crecimiento de los costes directos e indirectos ${ }^{2}$.

Las razones del incumplimiento de las citas programadas son complejas y sus causas precisan un análisis exhaustivo para poder introducir en su gestión aquellas medidas correctoras encaminadas a minimizar el problema.

Este hecho, ni es nuevo, ni afecta solo al sistema sanitario español. Diversos estudios $^{3-7}$ en países desarrollados presentan cifras de absentismo que varían entre el 5 al 25\%, datos similares a los arrojados por estudios españoles ${ }^{1,2,8-10}$ que oscilan entre el 10 y el $20 \%$.

Existen varias revisiones bibliográficas que evalúan los factores determinantes del absentismo de consultas, tanto en el entorno hospitalario como en atención primaria $^{11-13}$. Car y col llevan a cabo una revisión ${ }^{14}$ en la que analizan dichos factores en una amplia gama de entornos de países de Europa, Sudamérica y Sureste Asiático. Los estudios identifican como principales factores relacionados con el paciente para no cumplir con sus citas: el olvido, la confusión sobre la fecha/hora de la cita, la resolución de los síntomas, las creencias de salud, la falta y dificultad de transporte, los problemas de programación, el estado de salud, la resistencia a la consulta, y la frustración con la organización manifestada por largos tiempos de espera y discontinuidad de la atención. Entre los factores relacionados con el sistema destacan: la falta de comunicación entre los profesionales sanitarios y pacientes, los tiempos de espera, la calidad de consulta, la demora en la asignación de la cita, los problemas administrativos y/o de oficina y el lugar de la atención sanitaria.

La falta de asistencia a las citas programadas ha sido presentada como problemática en términos de pérdida de tiempo y el costo financiero involucrado ${ }^{15}$, a pesar de ello y aunque son muchos los autores que hacen referencia a este problema $^{1,3-6,10,13-17}$ han sido muy escasos los estudios que han evaluado mediante un análisis económico el impacto del absentismo de los pacientes en consultas externas en sus organizaciones ${ }^{2,4,15}$.

Para mejorar esta situación se pueden realizar dos enfoques: el análisis simple del absentismo de forma aislada con el objetivo de mejorar las tasas de asistencia y, el estudio completo del problema general del acceso a la salud y la demanda del paciente, explorando nuevas formas de trabajo mediante un enfoque centrado en el enfermo.

El objetivo del estudio fue identificar los factores de la persona, el proceso y el contexto que influyen en el absentismo en las citas de Consultas Externas en la Agencia Sanitaria Costa del Sol (ASCS), describiendo el perfil del paciente que no acude a su cita, los motivos de su absentismo y realizando una estimación del coste económico.

\section{MÉTODO}

Se realizó un estudio de casos y controles multicéntrico en los centros pertenecientes a la ASCS: hospital Costa del Sol, Centro de Alta Resolución de Especialidades de Mijas y hospital de Alta Resolución de Benalmádena en el que los casos estaban constituidos por una muestra de pacientes consecutivos de cada 
uno de los centros a los que pertenecían los pacientes que no acudieron a su cita programada de primera visita procedente de atención primaria o revisión y los controles una muestra de pacientes consecutivos de cada uno de los centros de los pacientes que sí acudieron a las citas anteriormente referidas en el mismo día y franja horaria, apareados por centro, servicio y tipo de consulta, durante el segundo semestre del año 2013 y el primero del 2014. Así mismo, se realizó un análisis de costes por centro de las citas de cada una de las especialidades en base a la contabilidad analítica, con una comparativa por centros de 2012.

Los sujetos de estudio fueron todos los pacientes mayores de 17 años, citados en primera visita médica procedente de atención primaria o de revisiones programadas derivadas de primeras visitas médicas, con capacidad para realizar encuesta telefónica y que dieron su consentimiento verbal para participar en el estudio. Se excluyeron aquellas citas de pacientes que no residían en el área de cobertura del hospital de referencia.

El proyecto obtuvo el visto bueno del Comité de Ética de Investigación Costa del Sol.

A partir de los datos de referencia del estudio de Morera-Guitart y col ${ }^{19}$, esperando una proporción del $30 \%$ de pertenencia a ámbito rural (o distancia superior de 25 $\mathrm{km}$ al centro de referencia) entre los sujetos identificados como casos (no acuden a cita), y del $15 \%$ en el mismo factor entre los sujetos identificados como controles (acuden a cita), para una potencia del $80 \%$, y un nivel de confianza del $95 \%$, planteando una selección 1: 2 (2 controles por caso incluido), el tamaño de muestra necesario fue de 98 casos y 196 controles (294 sujetos en total). La muestra se multiplicó por tres, ya que fueron tres los centros que se incluyeron.

Los sujetos del grupo Caso (no acuden a su cita) fueron incluidos en el estudio de forma consecutiva conforme se arrancó el periodo de recogida de datos, y los pacientes pertenecientes al grupo Control (acuden a su cita) se extrajeron del programa de citación HCIS entre los dos primeros sujetos del mismo día y franja horaria que el paciente Caso, apareados igualmente por centro, servicio y tipo de consulta. Posteriormente se efectuó el análisis de todas aquellas variables extraíbles de la aplicación informática de ambos grupos. Se realizó a continuación una entrevista telefónica por personal entrenado para la recogida del resto de los datos de ambas muestras, procediendo posteriormente al análisis comparativo de los datos obtenidos.

A los pacientes que no contestaron en la primera llamada se les efectuaron dos llamadas más en horarios y días diferentes; aquellos sujetos que no respondieron finalmente a la llamada telefónica, o que rechazaron participar en el estudio fueron sustituidos por el siguiente candidato del centro. Todas las encuestas fueron contestadas por los pacientes.

Las variables analizadas fueron de tipo sociodemográfico y contextuales de los pacientes: edad, sexo, nivel de estudios, autopercepción de salud, nacionalidad, percepción de ser atendido, pluripatología (más de dos enfermedades de base), ocupación, baja laboral, cargas familiares, dependencia del paciente, medio de transporte habitual utilizado cuando acude al centro hospitalario, frecuencia de citas de consultas externas en el último año, frecuencia de urgencias en los últimos 6 meses. También se estudiaron variables relativas al sistema y procesos de atención: tipo de consulta, especialidad médica de la consulta, tipo de aviso, demora en la asignación de la cita, distancia geográfica. En los sujetos etiquetados como casos también se evaluó el motivo de absentismo ${ }^{2}$ clasificándolos en dos grandes grupos de causas: evitables (olvido, error administrativo, fallo en la comunicación, pendiente de pruebas, insatisfacción) y no evitables (incapacidad física, mejoría, cambio de domicilio, otros motivos). Por último, se evaluaron variables de inputs en costes: coste unitario por centro y especialidad, extraídos de la contabilidad analítica de la base de datos de la Consejería de Salud de Andalucía. Los datos se expresan en 
costes totales que incluyen la imputación directa de costes controlables (personal, fungible y equipamiento, fármacos, consumos y prestaciones) a centros de responsabilidad por número de consultas externas (pacientes) y la imputación indirecta de costes de servicios (lavandería, limpieza, cocina, seguridad, mantenimiento, administración, informática...) mediante criterios de reparto (superficie útil, kilos de ropa lavada, $\mathrm{n}^{\circ}$ de dietas, $\mathrm{n}^{\circ}$ de profesionales...)

Las fuentes de datos utilizadas fueron la historia clínica hospitalaria informatizada y la entrevista domiciliaria y la base de datos de contabilidad analítica del Servicio Andaluz de Salud (COAN).

Para el estudio estadístico se realizó un análisis descriptivo usando medidas de tendencia central y de dispersión para variables cuantitativas, y distribución de frecuencias para las cualitativas, tanto para las variables independientes referentes a toda la muestra (casos y controles), como las variables exclusivas de la muestra de los casos. Posteriormente, se hizo un análisis bivariado tomando como variable principal ser caso o control ("no acuden" frente a los que "acuden"), para la comparación de subgrupos, utilizándose para las variables continuas la t-Student para muestras independientes (en el caso de distribución normal comprobado con test de Kolmogorov-Smirnov) y el test de la U de Mann-Whitney (en caso de distribución no normal); y en cuanto a las variables cualitativas o categóricas se utilizó el test de la Ji-Cuadrado, o de Fisher, en su caso (observaciones esperadas por casilla inferior a 5), para las variables cualitativas. Finalmente, se realizó un análisis de regresión logística múltiple, por pasos hacia delante, calculando las Odds Ratio (OR) y respectivos intervalos de confianza al $95 \%$.

\section{RESULTADOS}

Se recogieron 294 casos de pacientes que no asistieron a la cita (casos) y 588 controles. El $2 \%$ de los casos y $3 \%$ de los controles renunciaron a participar en el estudio, siendo sustituidos por el siguiente candidato del centro.

No se hallaron diferencias en la distribución de las características del centro y tipo de consulta entre grupo de casos y controles, salvo en la demora de asignación de cita, que era superior en los casos (media: 25, 2 días; DE: 37,3) que en los controles (media: 19,2; DE: 30,9). En ambos grupos la primera visita fue el tipo de consulta más valorado ( $48,6 \%)$, seguido de la primera revisión (por encima del 14\%) (Tabla 1).

Respecto a las características del paciente, en ambos grupos predominaban las citas de mujeres (más del 60\%), y la nacionalidad española (superior al 85\%). Se hallaron diferencias estadísticamente significativas en la edad, siendo superior los controles (media: 55,7; DE: 16,9) que en los casos (media: 51,3; DE: 17,5) (Tabla 1). No se encontraron diferencias entre ambos grupos en función de la especialidad a la que estaba asignada la consulta.

Los datos muestran que el análisis de tener familiares a cargo se encuentra al límite de la significación estadística $(\mathrm{p}=0,050)$, lo que supondría un factor de riesgo en el absentismo de los pacientes en las consultas externas. Pero al analizar si el tipo de carga familiar influye en el absentismo en consultas observamos que no es significativo $(p=0,201)$. Los hijos son el tipo de carga familiar más habitual tanto en los casos con un $93,9 \%(n=139)$, como en los controles con un 88,5\% ( $n=231)$ (Tabla 1).

En la información referente a uso de servicios sanitarios en el año previo, se hallaron diferencias significativas tanto en el número medio de veces que acudió a consultas externas, superior en los controles $(4,8$; DE: 5,0$)$ que en los casos $(3,6$; DE: 4,0$)$; como en la valoración cualitativa de la misma variable, dado que la presencia de acudir a consulta externa en el año previa era del $97,4 \%$ en los controles, frente al $78,2 \%$ en los casos (Tabla 2). 
Tabla 1. Características del centro, tipo de consulta y sujetos incluidos

Características del centro y tipo de consulta

\begin{tabular}{|c|c|c|c|c|c|}
\hline & \multicolumn{2}{|c|}{ Control } & \multicolumn{2}{|c|}{ Caso } & \multirow{2}{*}{$\mathbf{p}$} \\
\hline & $\mathrm{n}$ & $\%$ & $\mathrm{n}$ & $\%$ & \\
\hline \multicolumn{6}{|l|}{ Centro } \\
\hline CARE Mijas & 196 & 33,3 & 98 & 33,3 & \\
\hline HAR Benalmádena & 196 & 33,3 & 98 & 33,3 & \\
\hline HCS Marbella & 196 & 33,3 & 98 & 33,3 & \\
\hline \multicolumn{6}{|l|}{ Especialidad Médica } \\
\hline Anestesia & 18 & 3,1 & 9 & 3,1 & \\
\hline Cardiología & 54 & 9,2 & 27 & 9,2 & \\
\hline Cirugía & 56 & 9,5 & 28 & 9,5 & \\
\hline Dermatología & 28 & 4,8 & 14 & 4,8 & \\
\hline Digestivo & 50 & 8,5 & 25 & 8,5 & \\
\hline Ginecología & 42 & 7,1 & 21 & 7,1 & \\
\hline Hematología & 8 & 1,4 & 4 & 1,4 & \\
\hline Medicina Interna & 48 & 8,2 & 24 & 8,2 & \\
\hline Neumología & 48 & 8,2 & 24 & 8,2 & \\
\hline Oftalmología & 32 & 5,4 & 16 & 5,4 & \\
\hline Otorrinolaringología & 52 & 8,8 & 26 & 8,8 & \\
\hline Rehabilitación & 24 & 4,1 & 12 & 4,1 & \\
\hline Tocología & 42 & 7,1 & 21 & 7,1 & \\
\hline Traumatología & 54 & 9,2 & 27 & 9,2 & \\
\hline Urología & 32 & 5,4 & 16 & 5,4 & \\
\hline \multicolumn{6}{|l|}{ Tipo de consulta } \\
\hline Primera visita & 286 & 48,6 & 143 & 48,6 & \multirow{7}{*}{0,27} \\
\hline Revisión 1 mes & 98 & 16,7 & 43 & 14,6 & \\
\hline Revisión 2 meses & 56 & 9,5 & 20 & 6,8 & \\
\hline Revisión 3 meses & 48 & 8,2 & 30 & 10,2 & \\
\hline Revisión 4 meses & 15 & 2,6 & 14 & 4,8 & \\
\hline Revisión 6 meses & 59 & 10,0 & 35 & 11,9 & \\
\hline Revisión 1 año & 26 & 4,4 & 9 & 3,1 & \\
\hline \multicolumn{6}{|c|}{ Comunicación de la cita } \\
\hline Carta & 109 & 18,5 & 65 & 22,6 & \multirow{4}{*}{0,408} \\
\hline Personal & 230 & 39,1 & 119 & 41,3 & \\
\hline Teléfono & 232 & 39,5 & 99 & 34,4 & \\
\hline Dos vías & 17 & 2,9 & 5 & 1,7 & \\
\hline \multicolumn{6}{|c|}{ Considera que el motivo de acudir a cita precisa asistencia } \\
\hline Normal & 451 & 76,8 & 232 & 78,9 & \multirow{3}{*}{0,663} \\
\hline Preferente & 104 & 17,7 & 45 & 15,3 & \\
\hline Urgente & 32 & 5,5 & 17 & 5,8 & \\
\hline \multicolumn{6}{|c|}{ Demora Asignación de cita (días) } \\
\hline Media; DE & 19,2 & 30,9 & 25,2 & 37,3 & 0,048 \\
\hline
\end{tabular}




\begin{tabular}{|c|c|c|c|c|c|c|}
\hline \multicolumn{7}{|c|}{ Características de los sujetos incluidos } \\
\hline & & \multicolumn{2}{|c|}{ Control } & \multicolumn{2}{|c|}{ Caso } & \multirow{2}{*}{$\mathbf{p}$} \\
\hline & & $\mathrm{n}$ & $\%$ & $\mathrm{n}$ & $\%$ & \\
\hline \multirow{2}{*}{ Sexo } & Hombre & 209 & 35,5 & 122 & 37,5 & \multirow{2}{*}{0,059} \\
\hline & Mujer & 379 & 64,5 & 172 & 62,5 & \\
\hline Edad & Media & 55,7 & & 51,3 & & $<0,001$ \\
\hline \multirow{2}{*}{ Nacionalidad } & Española & 532 & 90,5 & 254 & 86,4 & \multirow{2}{*}{0,085} \\
\hline & Extranjera & 56 & 9,5 & 40 & 13,6 & \\
\hline \multirow{2}{*}{$\begin{array}{l}\text { Distancia geográfica al } \\
\text { centro }\end{array}$} & $<10 \mathrm{kms}$ & 411 & 69,9 & 216 & 73,5 & \multirow{2}{*}{0,436} \\
\hline & $>=10 \mathrm{kms}$ & 177 & 30,1 & 78 & 26,5 & \\
\hline \multirow{4}{*}{$\begin{array}{l}\text { Transporte habitual } \\
\text { acudir a centro }\end{array}$} & A pie & 60 & 10,2 & 35 & 11,9 & \multirow{4}{*}{0,752} \\
\hline & Transporte público & 91 & 15,5 & 38 & 12,9 & \\
\hline & Vehículo propio & 328 & 55,8 & 172 & 58,5 & \\
\hline & Vehículo de amigo o familiar & 109 & 18,5 & 49 & 16,7 & \\
\hline \multirow{4}{*}{ Nivel de estudios } & Sin estudios & 103 & 17,5 & 42 & 14,3 & \multirow{4}{*}{0,437} \\
\hline & Primarios & 221 & 37,6 & 113 & 38,4 & \\
\hline & Medios & 174 & 29,6 & 99 & 33,7 & \\
\hline & Universitarios & 90 & 15,3 & 40 & 13,6 & \\
\hline \multirow{2}{*}{ Estado de salud percibido } & Muy bueno - Bueno & 369 & 62,8 & 176 & 60,1 & \multirow{2}{*}{0,538} \\
\hline & Regular - Malo - Muy Malo & 219 & 37,2 & 117 & 39,9 & \\
\hline \multirow{2}{*}{$\begin{array}{l}\text { Presencia de más de } \\
\text { dos enfermedades o } \\
\text { diagnósticos médicos }\end{array}$} & No & 321 & 54,6 & 176 & 60,1 & \multirow[b]{2}{*}{0,070} \\
\hline & Sí & 267 & 45,4 & 117 & 39,9 & \\
\hline \multirow{7}{*}{ Situación laboral } & Cuenta propia & 49 & 8,3 & 32 & 10,9 & \multirow{7}{*}{0,210} \\
\hline & Cuenta ajena & 187 & 31,8 & 103 & 35,0 & \\
\hline & Empresario & 1 & 0,2 & 0 & 0,0 & \\
\hline & Ama de casa & 98 & 16,7 & 38 & 12,9 & \\
\hline & Estudiante & 8 & 1,4 & 9 & 3,1 & \\
\hline & Pensionista & 177 & 30,1 & 75 & 25,5 & \\
\hline & Desempleado & 68 & 11,6 & 37 & 12,6 & \\
\hline \multirow{2}{*}{$\begin{array}{l}\text { Incapacidad laboral } \\
\text { transitoria }\end{array}$} & No & 558 & 95,1 & 287 & 97,6 & \multirow{2}{*}{0,066} \\
\hline & Sí & 29 & 4,9 & 7 & 2,4 & \\
\hline \multirow{2}{*}{ Familiar a cargo } & No & 328 & 55,9 & 146 & 49,7 & \multirow{2}{*}{0,05} \\
\hline & Sí & 260 & 44,3 & 148 & 50,3 & \\
\hline \multirow{2}{*}{ Hijos a cargo } & No & 357 & 60,7 & 155 & 52,7 & 0201 \\
\hline & Sí & 231 & 39,3 & 139 & 47,3 & 0,201 \\
\hline Cuidado de personas & No & 561 & 95,4 & 285 & 96,9 & ביב 0 \\
\hline mayores & Sí & 27 & 4,6 & 9 & 3,1 & 0,201 \\
\hline & Total & 3 & 0,5 & 0 & 0,0 & \\
\hline & Severa & 2 & 0,3 & 2 & 0,7 & \\
\hline Nivel de dependencia & Moderada & 20 & 3,4 & 15 & 5,1 & 0,300 \\
\hline & Escasa & 50 & 8,5 & 18 & 6,1 & \\
\hline & Independiente & 512 & 87,2 & 258 & 88,1 & \\
\hline
\end{tabular}


Tabla 2. Causas de absentismo y frecuentación de servicios en 6 últimos meses

\begin{tabular}{|c|c|c|c|c|c|}
\hline & \multicolumn{2}{|c|}{ Control } & \multicolumn{2}{|c|}{ Caso } & \multirow{2}{*}{$\mathbf{p}$} \\
\hline & $\mathrm{n}$ & $\%$ & $\mathrm{n}$ & $\%$ & \\
\hline \multicolumn{6}{|c|}{ Por qué no acudió a su cita } \\
\hline Olvido & & & 87 & 29,7 & \\
\hline Error administrativo & & & 10 & 3,4 & \\
\hline Fallo comunicación & & & 47 & 16,0 & \\
\hline Mejoría & & & 14 & 4,8 & \\
\hline Incapacidad física & & & 11 & 3,8 & \\
\hline Prueba pendiente & & & 3 & 1,0 & \\
\hline Insatisfacción & & & 6 & 2,0 & \\
\hline Otros motivos* & & & 115 & 39,3 & \\
\hline \multicolumn{6}{|l|}{ Nueva cita } \\
\hline No & & & 124 & 42,2 & \\
\hline Sí & & & 170 & 57,8 & \\
\hline \multicolumn{6}{|c|}{ Cuántas veces acude a urgencias en últimos 6 meses } \\
\hline Media & 0,6 & & 0,8 & & 0,183 \\
\hline \multicolumn{6}{|c|}{ Cuántas veces acude a consultas externas en último año } \\
\hline Media & 4,8 & & 3,6 & & $<0,001$ \\
\hline \multicolumn{6}{|c|}{ Acude a urgencias en últimos 6 meses } \\
\hline No & 364 & 61,9 & 172 & 58,5 & \multirow{2}{*}{0,367} \\
\hline Sí & 224 & 38,1 & 122 & 41,5 & \\
\hline \multicolumn{6}{|c|}{ Acude a consultas externas en último año } \\
\hline No & 15 & 2,6 & 64 & 21,8 & \multirow{2}{*}{$<0,001$} \\
\hline Sí & 573 & 97,4 & 230 & 78,2 & \\
\hline
\end{tabular}

*Viajes, motivos laborales, confusión en la cita, problemas de transporte, hospitalización, otras enfermedades, visita a médico privado, otra cita médica el mismo día, accidente de tráfico.

De la entrevista específica a los casos sobre los motivos de no acudir a la cita, el $29,7 \%$ reflejaron el olvido y un $16 \%$ fallo en la comunicación entre sistema y paciente. Un total de 170 casos $(57,8 \%)$ solicitaron una nueva cita (Tabla 2).

Se diseñó un modelo de regresión logística multivariante, tomando como variable dependiente la presencia o ausencia de absentismo en las consultas y como predictoras, aquellas que en el análisis bivariante habían mostrado algún indicio de influencia o asociación. El modelo final, presentó un ajuste adecuado (prueba de Hosmer-Lemeshow, $\mathrm{p}=0,151$ ), con una cla- sificación adecuada del $68,3 \%$. El modelo mostró cómo la demora en la cita actuaba como factor protector en el caso de que ésta fuera menor; que la edad se asocia inversamente al absentismo en consultas y que la frecuentación habitual a consultas en el último año actúa también como factor protector del absentismo a éstas (Tabla 3).

El impacto económico total en la ASCS fue superior a 3 millones de euros para una tasa de inasistencia del $13,8 \%$, representando un $14 \%$ del total del global de las consultas de la agencia (400.761 citas). Por centros, el coste económico en el Hospital Costa del sol ascendió a 2.475.640 € con un 
Tabla 3. Regresión logística multivariante de riesgo de ser caso

\begin{tabular}{lccccc} 
& Variables & $\boldsymbol{\beta}$ & $\mathbf{p}$ & Odds Ratio & \multicolumn{2}{c}{ IC 95\% } \\
\cline { 4 - 7 } & & & & Inferior & Superior \\
\hline Edad & 0,015 & 0,001 & 1,015 & 1,006 & 1,024 \\
\hline Sexo & $-0,285$ & 0,059 & 0,752 & 0,56 & 1,01 \\
\hline Baja laboral & 0,801 & 0,066 & 2,228 & 0,949 & 5,227 \\
\hline Demora citas (días) & $-0,005$ & 0,019 & 0,995 & 0,991 & 0,999 \\
\hline Acude a consultas & 0,052 & 0,001 & 1,053 & 1,013 & 1,096 \\
\hline
\end{tabular}

Potencia estadística para la OR de edad, demora en la cita y frecuentación consultas: $99,7 \%$

incumplimiento del $14,2 \%$, en el Hospital de Alta Resolución de Benalmádena fue de $515.936 €$ con el $12,2 \%$ y en el Centro de Alta Resolución de Mijas se registró un coste de $395.342 €$ con una tasa de inasistencia del $13,5 \%$ (Fig. 1).

\section{DISCUSIÓN}

Los resultados obtenidos ponen de relieve que sujetos jóvenes con demora en la adjudicación de la cita y sin antecedentes de frecuentación de consultas es el perfil del absentista en nuestra área sanitaria, siendo el olvido y el fallo en la comunicación los principales motivos manifestados por los pacientes para no acudir a su consulta.

En relación al sexo, nuestros datos se encuentran al límite de la significación, al igual que estar de baja laboral, aunque ésta con menor significación. En la literatura, la relación entre el género y el absentismo en consultas no aparece de forma concluyente, si bien, las referencias en cuanto a una mayor frecuencia en varones son superiores ${ }^{2,6,8,10}$.

Negro et col afirman, en base a estudios previos, que no existe un perfil definido

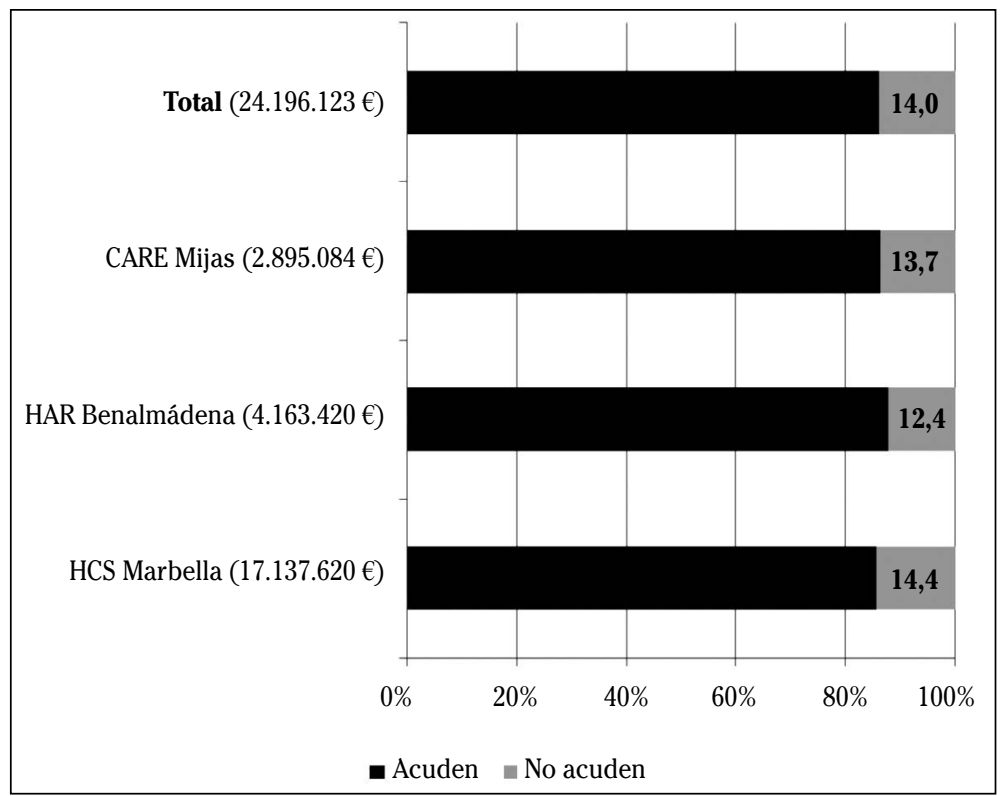

Figura 1. Estimación de costes. 
del paciente incumplidor en general, pero sí son muchos los autores que han hallado datos que ponen de manifiesto, como en nuestro caso, un mayor incumplimiento en pacientes jóvenes $\mathbf{s}^{1,3,6,8,10,11,18,19}$, explicado quizás por la percepción de salud de este grupo de edad.

Un dato muy a tener en cuenta es la demora en la asignación de la cita como factor contribuyente. Ya en 1996 Bowman y col mostraron que un menor tiempo de espera mejora la tasa de asistencia en primeras visitas ${ }^{20}$. En este sentido, nuestro estudio confirma resultados previos de que la demora en la cita en general es un factor de riesgo para el absentismo de los usuarios a las consultas externas ${ }^{12,15,21,22}$.

No hemos encontrado diferencias estadísticamente significativas en referencia a la lejanía al centro, aunque existen varios estudios de nuestro entorno ${ }^{8,9}$ que las avalan, dato éste explicado por la buena accesibilidad (distancia media inferior a $10 \mathrm{Km}$ ) a los centros de nuestra área de cobertura. Tampoco ha sido determinante el transporte utilizado por el paciente para trasladarse al hospital, hecho que parece haber influido en otros estudios, generalmente en contextos asistenciales con desarrollo deficitario $0^{5,7}$.

Los datos muestran diferencias estadísticamente significativas en cuanto a la frecuentación previa a consultas externas, actuando ésta como un factor protector. La bibliografía de referencia no analiza este factor, pero son muchos los autores que han relacionado que los pacientes que no acudieron a su cita médica, habían presentado incumplimiento previo ${ }^{3,4,8,10}$, por lo que manifiestan un perfil de paciente reincidente. Así mismo, el 57,8\% de los pacientes que faltaron a su cita solicitaron una nueva cita, cifra muy similar a la obtenida por Neal y col (60\%) en el contexto de consultas de medicina general en West Yorkshire (Reino Unido) ${ }^{3}$. Esto nos permite hacer una doble lectura: el incremento del coste sanitario imputado y la elección de otras opciones antes que asistir a la consulta médica (viajes, motivos laborales). Puede también influir el agravamiento de su enfermedad o la interferencia de otros problemas de salud (hospitalización, otras enfermedades), aunque en menor medida. No puede descartarse tampoco, aunque en este estudio es imposible de discernir, que haya existido una inadecuación en la indicación de la derivación a consultas de atención especializada.

La relevancia del estudio de los fallos en las citas viene dada por el hecho de que la mayoría de las causas por las que los pacientes no se presentan a sus citas podrían evitarse $^{9}$. Varios estudios que han analizado las causas de la inasistencia cifran los motivos evitables en torno al $56 \%^{9,23}$, dato muy aproximado al 52,4\% que hemos hallado en nuestro análisis. El principal motivo evitable ha sido el olvido y muchos estudios avalan este dato ${ }^{1,3,4,7-9,24-26}$, cuya cifra varía desde el 13,7\% hallado por Fonseca y col en el ámbito hospitalario en nuestro país1, hasta el $40 \%$ de Neal y col en el contexto ambulatorio en Reino Unido ${ }^{3}$. Diversas intervenciones utilizando diferentes métodos de aviso han sido probadas para reducir la tasa de fallos en las citas ${ }^{19}$. La mayoría de los estudios han demostrado una reducción en las tasas de inasistencia, independientemente del método de recordatorio utilizado ${ }^{16,27}$. Los recordatorios postales son eficaces, pero costosos, y su efecto disminuye con el tiempo ${ }^{4,28}$. En cuanto a los recordatorios telefónicos, varios ensayos clínicos mostraron evidencia de que estos pueden reducir las citas perdidas. Sawyer y col obtuvieron una reducción de la tasa de inasistencia de un $12 \%$ en adolescentes ${ }^{24} \mathrm{y}$ Hashim et al del $7 \%$ en población urbana neoyorquina ${ }^{29}$. En referencia a los mensajes de texto, Liew et al concluyen que son igual de efectivos que los recordatorios telefóni$\cos ^{26}$, y Gurol-Urganci y col en revisión sistemática de 2013 manifiestan que son más efectivos que ningún recordatorio ${ }^{25}$.

Varios estudios apuntan a este método como el más coste-efectivo ${ }^{19,30}$ y Hasvold y col concluyen tras su revisión sistemática, que el coste de su implantación en el sistema sería menor al que soporta la organización por la inasistencia de nuestros pacientes a las consultas médicas ${ }^{30}$. Si bien hay que tener en cuenta que el éxito de la implantación de este sistema pasa por tener 
una base de datos de teléfonos de contacto de los pacientes actualizada.

El fallo de comunicación fue la segunda causa más frecuente (16\%) de error en la cita por causas evitables, hallazgo que no coincide con Morena-Guitard y col que lo sitúan en quinto lugar ${ }^{9}$. Es necesario en este sentido implementar medidas de mejora encaminadas a la asignación de la cita de revisión en el acto de la consulta médica, con lo que el paciente tiene la opción de elegir el momento de su cita programada permitiendo la conciliación con su vida laboral y personal. Para ello es imprescindible mejorar la gestión de las agendas realizando una programación anual que posibilite la asignación de revisiones de larga data.

Casi la mitad de los motivos correspondería en este caso a causas no evitables tales como mejoría e incapacidad física, otras prioridades $\mathrm{u}$ otros motivos (laborales, personales). Estos datos ponen de manifiesto la importancia de desarrollar intervenciones no solo encaminadas a paliar y corregir el impacto de los motivos evitables sino a concienciar a la ciudadanía de la necesidad de en caso de que no pueda acudir a su cita programada, avise para que se pueda citar en su lugar a otro paciente. Una medida a valorar en este caso sería la disponibilidad de un número directo de contacto del centro para anular las citas que constara impreso en la nota de cita del paciente.

Las citas perdidas son una de las principales causas de la ineficiencia en la prestación de asistencia sanitaria, con importantes costes monetarios para los sistemas de salud, y retrasos en el diagnóstico y el tratamiento para el paciente que no asiste ${ }^{14}$. En nuestro país, varios son los estudios que hacen referencia al coste que supone la inasistencia para el sistema sanitario $^{1,2,8,10}$ pero solo dos aportan datos económicos del mismo. Negro y col cuantifican el coste de las citas perdidas durante un año en el servicio de Alergología en 109 mil euros ${ }^{8}$ y Alcolea y col cifran en 14 mil euros el coste de la inasistencia a consultas durante un periodo de cinco meses en los Hospitales del Consorcio Aragonés de Alta Resolución ${ }^{2}$.
En esta línea, nuestro análisis constata el gasto total en $3.386 .919 €$ para una tasa de inasistencia del 13,8\%, representando un $14 \%$ del total del coste global de las consultas de la ASCS. Estos datos ponen de manifiesto la relevancia del desarrollo de medidas encaminadas a reducir las cifras de incumplimiento en citas médicas en las organizaciones sanitarias.

El estudio presenta algunas limitaciones derivadas del posible sesgo de no respuesta a las llamadas para las encuestas, dado que los sujetos que no acuden a su cita podían tener una menor motivación a no participar en el estudio, o a no contestar al teléfono. No obstante, la tasa de no respuesta ( $2 \%$ en los casos y $3 \%$ en los controles) no representa diferencias significativas entre ambos grupos, y tampoco hubo ninguna pérdida por fallecimiento.

Así mismo, no se estudiaron las causas por las que los pacientes que no acudieron a su visita médica no solicitaron una nueva cita, lo que nos habría permitido valorar aspectos como la indicación médica en el caso de que el motivo hubiera sido "porque no la necesitaran".

\section{BIBLIOGRAFÍA}

1. Fonseca E, Vázeuez P, Mata P, Pita S, Muiño MS. Estudio de la inasistencia a las citaciones en consulta en un servicio de dermatología. Piel 2001; 16: 485-489.

2. Alcolea M, Gorgemans S. Absentismo de pacientes citados en las consultas de Atención Especializada en el Consorcio Aragonés Sanitario de Alta Resolución: repercusiones económicas y demoras. Rev Adm Sanit 2009; 7: 715-728.

3. Neal RD, Hussain-Gambles M, Allgar VL, Lawlor DA, DEMPSEY O. Reasons for and consequences of missed appointments in general practice in the UK: questionnaire survey and prospective review of medical records. BMC Fam Pract 2005; 6: 47.

4. Murdock A, Rodgers C, Lindsay H, Tham TC. Why do patients not keep their appointments? Prospective study in a gastroenterology outpatient clinic. J R Soc Med 2002; 95: 284-286.

5. VAN DER MEer G, LOOCK JW. Why patients miss follow-up appointments: a prospective control-matched study. Afr J Public Health 2008; 5: 154-156. 
6. ZiRKLE MS, McNelles LR. Nonattendance at a hospital-based otolaryngology clinic: a preliminary analysis within a universal healthcare system. Ear Nose Throat J 2011; 90: E32E34.

7. Nour El-Din MM, Al-ShaKhs FN, Al-Oudah SS. Missed appointments at a university hospital in eastern Saudi Arabia: magnitude and association factors. J Egypt Public Health Assoc 2008; 83: 415-433.

8. Negro JM, Campuzano FJ, Pellicer F, Bernal T, Martos MD, Martínez R et al. Incumplimiento de citas concertadas en los pacientes de una consulta de alergología tras el segundo año de implantación de un ciclo de mejora. Alergol Inmunol Clin 2004; 19: 195-204.

9. Morera-Guitard J, Mas-Server MA, Más-Sese J. Análisis de los pacientes no presentados a la consulta de neurología de la Marina Alta. Rev Neurol 2002; 34: 701-705.

10. Orueta R, Gómez-Calcerrada RM, Redondo S, Soto M, Alejandre G, López J. Factores relacionados con el incumplimiento a citas concertadas de un grupo de pacientes hipertensos. MEDIFAM 2001; 11: 140-146.

11. Deyo RA, Inur TS. Dropouts and broken appointments. A literature review and agenda for future research. Medical Care 1980; 18: 1146-1157.

12. Sharp DJ, Hamilton W. Non-attendance at general practices and outpatient clinics. BMJ 2001; 323: 1081-1082.

13. George A, Rubin G. Non-attendance in general practice: a systematic review and its implications for access to primary health care. Family Practice 2003; 20: 178-184.

14. Car J, Gurol-Urganci I, De Jongh T, Vodopivec JAMSEK-V, R ATun. Mobile phone messaging reminders for attendance at healthcare appointments. Cochrane Data-base Syst Rev 2012; 7: CD007458.

15. Martin C, Perfect T, Manto G. Non-attendance in primary care: the views of patients and practices on its causes, impact and solutions. Fam Pract 2005; 22: 638-643.

16. Stubbs ND, Geraci SA, Stephenson PL, Jones DB, SANDERS S. Methods to reduce outpatient nonattendance. Am J Med Sci 2012; 344: 211-219.

17. Negro Álvarez JM, Félix Toledo R, Guerrero FernÁndez M, Campuzano López FJ, Bernal Fernández T, Pellicer F et al. Evaluación de los resultados obtenidos en el incumplimiento de citas concertadas en pacientes de una consulta de Alergología, al año de la implantación de un ciclo de mejora. Gestión Hospitalaria 2004; 15: 13-22.
18. Giunta D, Briatore A, Baum A, Luna D, Waisman G, DE Quiros FG. Factors associated with nonattendance at clinical medicine scheduled outpatient appointments in a university general hospital. Patient Prefer Adherence 2013; 7: 1163-1170.

19. Perron NJ, Dao MD, Kossovsky MP, Miserez V, Chuard C, Calmy A, Gaspoz JM. Reduction of missed appointments at an urban primary care clinic: a randomised controlled study. BMC Fam Pract 2010; 11: 79.

20. Bowman RJC, Bennett HGB, Houston CA, AitCHENSON TC, DutTon GN. Waiting times for and attendance at paediatric ophthalmology outpatient appointments. BMJ 1996; 313: 1244.

21. BICKLER CB. Defaulted appointments in general practice. J R Coll Gen Pract 1985; 35: 19-22.

22. HAGERMAN GA. Testing the mailed appointment reminder in family practice. J Fam Pract 1978; 7: 199-201.

23. Sañudo S, González JM, Gamazo JC, Albillos ML, Gayubo P, De Uribe F. Pacientes que no acuden a consulta: características, repercusiones económicas y de gestión, y posibles soluciones. Soria: Actas del VII congreso de Economía Regional de Castilla y León; 2003.

24. SAWyer SM, Zalan A, Bond LM. SAWyer SM, Zalan A, Bond LM. Telephone reminders improve adolescent clinic attendance: a randomized controlled trial. J Paediatr Child Health 2002; 13: 79-83.

25. Gurol-Urganci I1, DE Jongh T, Vodopivec-JamseK V, ATUN R, CAR J. Mobile phone messaging reminders for attendance at healthcare appointments. Cochrane Data-base Syst Rev $2013 ; 12$.

26. Liew SM, Tong SF, Lee VK, NG CJ, Leong KC, Teng CL. Text messaging reminders to reduce nonattendance in chronic disease follow-up: a clinical trial. Br J Gen Pract 2009; 59: 916-920.

27. Koshy E, CAR J, Majeed A. Effectiveness of mobile-phone short message service (SMS) reminders for ophthalmology outpatient appointments: Observational study. BMC Ophthalmol 2008; 8: 9.

28. HENDERSOn R. Encouraging attendance at outpatient appointments: can we do more? Scott Med J 2008; 13: 9-12.

29. Hashim MJ, Franks P, Fiscella K. Effectiveness of telephone reminders in improving rate of appointments kept at an outpatient clinic: a randomized controlled trial. J Am Fam Pract Junta 2001; 13: 193-196.

30. Hasvold PE, Wootton R. Use of telephone and SMS reminders to improve attendance at hospital appointments: a systematic review. J Telemed Telecare 2011; 17: 358-364. 